\title{
A magnetodisc model service for planetary space weather studies
}

\author{
Nicholas Achilleos ${ }^{1}$, Patrick Guio ${ }^{1}$, Nicolas André ${ }^{2}$, and Arianna M. Sorba ${ }^{1}$ \\ ${ }^{1}$ Department of Physics and Astronomy/Centre for Planetary Sciences, University College London, Gower Street, WC1E 6BT London, UK \\ ${ }^{2}$ Institut de Recherche en Astrophysique et Planétologie (IRAP), Université de Toulouse, CNRS, CNES, UPS, 9 av. du colonel Roche, \\ BP 44346, 31028 Toulouse Cedex 4, France
}

Received 18 September 2018 / Accepted 22 May 2019

\begin{abstract}
Theoretical models play an important role in the Planetary Space Weather Services (PSWS) of the European Planetary Network ("Europlanet"), due to their ability to predict the physical response of magnetospheric environments to compressions or rarefactions in the upstream solar wind flow. We illustrate this aspect by presenting examples of some calculations done with the UCL Magnetodisc Model in both "Jupiter" and "Saturn" mode. Similar model outputs can now be provided via the PSWS MAGNETODISC service. For each planet's space environment, we present example model outputs showing the effect of compressions and rarefactions on the global magnetic field, plasma pressure and azimuthal current density. As a simple illustration of the physics underlying these reference models, we quantify solar wind effects by comparing the "compressed" and "expanded" outputs to a nominal "average-state" model, reflecting more typical solar wind dynamic pressures. We also describe the implementation of the corresponding PSWS MAGNETODISC Service, through which similar outputs may be obtained by potential users.
\end{abstract}

Keywords: magnetospheres / Jupiter / Saturn / magnetic fields / plasma

\section{Introduction}

This report describes the development of theoretical models for giant planet magnetodiscs, and online services which provide related model outputs for users' scientific studies. The relevant theoretical background is initially described in this section, and this is followed by sections which provide a description of some example model outputs, and the related online services.

We have used the method of Caudal (1986), later adapted by Achilleos et al. (2010a), to compute axisymmetric models of the rotating Jovian and Kronian plasmadiscs in which magnetic, centrifugal and plasma pressure forces are in equilibrium. The reader is referred to those studies for full details of the models used. To summarise briefly the essential method, the magnetic field $\boldsymbol{B}$ for the assumed-axisymmetric system (whose origin is at the planet centre) is expressed in terms of two Euler potentials, $\alpha$ and $\beta$ :

$$
\boldsymbol{B}=\nabla \alpha \times \nabla \beta
$$

In a spherical polar coordinate system, $\beta$ may be chosen to be only a function of the azimuthal angle $\phi$. For this choice, the poloidal field components, $B_{r}$ and $B_{\theta}$ (where $r$ and $\theta$ respec-

\footnotetext{
*Corresponding author: nicholas.achilleos@ucl.ac.uk
}

tively denote radial distance and co-latitude with respect to the magnetic/rotation axis) are given by:

$$
\begin{gathered}
B_{r}=\frac{a}{r^{2} \sin \theta} \frac{\partial \alpha}{\partial \theta}, \\
B_{\theta}=-\frac{a}{r \sin \theta} \frac{\partial \alpha}{\partial r},
\end{gathered}
$$

where $a$ denotes the radius of the central planet. Note that magnetic field lines lie along surfaces of constant $\alpha$, and the magnitude of the magnetic flux bounded between two neighbouring shells defined by the increment $\delta \alpha$ is simply $\left|\delta \Phi_{B}\right|=2 \pi a|\delta \alpha|$ (note that Achilleos et al., 2010a give an analogous expression in a dimensionless system of units).

Caudal (1986) demonstrated that the assumption of force equilibrium on the rotating plasma - i.e. the balance between the magnetic force, centrifugal force and plasma pressure gradient - was equivalent to a second-order partial differential equation describing the behaviour of the function $\alpha$. In order to solve this equation, and obtain a magnetostatic equilibrium solution for $\alpha$, one must adopt an "iterative" technique, initially assuming a pure dipole field. At each iteration, the magnetic field structure is used to re-compute the "plasma source function" which features in the differential equation and links the field structure with various properties of the rotating plasma. An updated solution for $\alpha$ is then obtained from the new source function and the procedure is repeated. 
The source function itself is constructed using the magnetic field model at any given iteration, along with observations of properties of the plasma over a convenient reference surface usually taken to be the equator. One commences this construction by using relatively simple parameterizations of spacecraft observations of the equatorial plasma temperature, flux tube content, angular velocity, and composition. We then combine these data-derived profiles with a magnetic field model to acquire the value of the source function throughout the magnetospheric model volume, under the force balance assumption. More details are in Caudal (1986) and Achilleos et al. (2010a), who also describe "averaging" methods that they apply to data to acquire realistic profiles for the axisymmetric model formalism.

The method of calculation of these magnetodisc solutions requires that various parameters of the plasma be initially specified on an appropriate reference surface - usually the equatorial plane. Reference values for plasma ion flux tube content, temperature, ion mass and angular velocity are acquired from appropriate spacecraft observations. For the energetic ("hot") particle population, the plasma pressure $P_{h}$ is computed through the use of a simplified equatorial profile of the product $P_{h} V_{\alpha}$, where $V_{\alpha}$ denotes unit flux tube volume. A convenient, although idealized, representation involves setting $P_{h} V_{\alpha}=K_{h}$ (constant) beyond a prescribed radial distance ( $L$ shell). As the calculation proceeds, plasma pressure values for both the thermal and hot plasma are re-computed according to the field structure acquired at each iteration. This aspect stabilises the approach towards the final solution, which is usually taken to be the iteration where the maximum relative change in the function value $\alpha$ falls below a prescribed tolerance. The calculation is usually performed on a fixed, uniformly spaced, circular grid in $r$ and $\mu(\mu=\cos \theta)$, with the maximum value of $r$ being referred to as $R_{\mathrm{mp}}$, the "standoff distance" of the dayside magnetopause. $R_{\mathrm{mp}}$ is a convenient parameter for the global size of the modelled system. The reader is referred to the aforementioned publications for further details.

Since these types of solutions represent a differentially rotating magnetosphere in equilibrium, it is also possible to use the total pressure value (plasma plus magnetic pressure), taken at the equatorial point of the prescribed outer boundary of the model, as a proxy for the dynamic pressure of the upstream solar wind (e.g., Sorba et al., 2017). This pressure value may be slightly modified by using an order-of-unity factor to account for the divergence of solar wind flow streamlines (Sorba et al., 2017). In the present study, our aim is to present a series of reference models for the magnetodiscs of both Jupiter and Saturn, corresponding to different values of $R_{\mathrm{mp}}$, in order to analyse the effects of changes in system size (or, equivalently, solar wind dynamic pressure) on the ensuing magnetospheric structure.

We describe these reference models in Section 2, and also comment therein on how model structure responds to changes in $R_{\mathrm{mp}}$. In this technical report, that same section serves as an illustration of the kind of model outputs for magnetodisc regions which can be obtained via the PSWS MAGNETODISC service. Section 3 contains a detailed description of this and other online services designed to provide magnetodisc model outputs for users, including model runs on request. We summarise our content in Section 4.

In a wider context, similar force-balance modelling has been described for the terrestrial system by Lackner (1970), and
Sozou \& Windle (1970) analysed the field structure associated with an intense ring current in the Earth's magnetosphere. There have also been numerous studies featuring ring current models for giant planet magnetospheres, based on a formalism by Connerney et al. (1981a) where the spatial dependence of the azimuthal current density is assumed a priori, and the corresponding field components then calculated (e.g., Connerney et al., 1981a, 1981b; Giampieri \& Dougherty, 2004; Bunce et al., 2007; Kellett et al., 2009).

\section{Reference magnetodisc models}

To illustrate the effects of solar wind dynamic pressure on magnetic field line structure, we calculated models for each planet with the "magnetopause radii" $R_{\mathrm{mp}}$ shown in Table 1, which can be considered equivalent to dayside magnetopause location ("standoff distance"). Similar model outputs (see Sect. 4) are readily available via the Europlanet Virtual European Solar and Planetary Access (VESPA) interface (http://www. europlanet-vespa.eu/, service "MDISC" - note that this VESPA service also provides basic thumbnail plots of field strength for the user). Table 1 also shows estimates of the solar wind dynamic pressure $P_{\mathrm{sw}}$ which correponds to equilibrium magnetopause states at both planets, according to the relations between $P_{\mathrm{sw}}$ and $R_{\mathrm{mp}}$ described by Pilkington et al. (2015) (Saturn "average" state magnetosphere), and Joy et al. (2002) (Jupiter).

\subsection{Saturn models}

We consider firstly the Saturn magnetodisc field models, for which we used a hot plasma index $K_{h}=2 \times 10^{6} \mathrm{~Pa} \mathrm{~m} \mathrm{~T}^{-1}$ for the calculations ("median" ring current state, see Achilleos et al., 2010b). The calculations were performed on a grid containing $N_{\mu}=501$ uniformly spaced points along the dimension corresponding to cosine of colatitude, $-1 \leq \cos \theta \leq 1$, and $N_{r}=200$ points along the dimension corresponding to radial distance, $1 \leq r \leq R_{\mathrm{mp}}$. Field lines for these models, on a single magnetic meridian, are shown in Figure 1, where the magnetic/ spin equator is defined by $Z=0$. The set of values of the magnetic potential $\alpha$ used to plot the field lines (contours of $\alpha$ ) is the same for all three configurations shown - hence, the field lines in each panel are anchored to the same set of ionospheric footpoints. The "sharpest" field lines with smallest equatorial radius of curvature are seen in the expanded, "disc-like" configuration, illustrating the role of magnetic curvature force in maintaining equilibrium of the outer magnetosphere. At the other extreme, the compressed, "dipole-like" field can maintain a given curvature force with less radial "stretching" of the field line, since the magnetic field strength is elevated for that configuration (see Achilleos et al., 2010a for more details).

The equatorial field strength profiles for these three configurations are shown in Figure 2. As expected, the compressed system displays the largest field strengths. All of the profiles, when compared to that of a pure dipole (appropriate for Saturn), show inner regions where the model field strength falls below the dipole, and outer regions where it exceeds the dipole field. This behaviour is a signature of the "solenoid-like" field generated by the magnetodisc current distribution. Furthermore, the 
Table 1. Magnetopause radii $\left(R_{\mathrm{mp}}\right)$ used for model calculations, with corresponding estimates of solar wind dynamic pressure $\left(P_{\mathrm{sw}}\right)$ (see text for sources).

\begin{tabular}{lccccc}
\hline Model field (Saturn) & $R_{\mathrm{mp}}($ Planet radii) & $P_{\mathrm{sw}}(\mathrm{nPa})$ & Model field (Jupiter) & $R_{\mathrm{mp}}(\mathrm{Planet}$ radii) & $P_{\mathrm{sw}}(\mathrm{nPa})$ \\
\hline Dipole-like & $15 R_{\mathrm{S}}$ & 0.400 & Quasi-dipolar & $60 R_{\mathrm{J}}$ & 0.39 \\
Average & $25 R_{\mathrm{S}}$ & 0.024 & Average & $75 R_{\mathrm{J}}$ & 0.13 \\
Disc-like & $35 R_{\mathrm{S}}$ & 0.004 & Disc-like & $90 R_{\mathrm{J}}$ \\
\hline
\end{tabular}

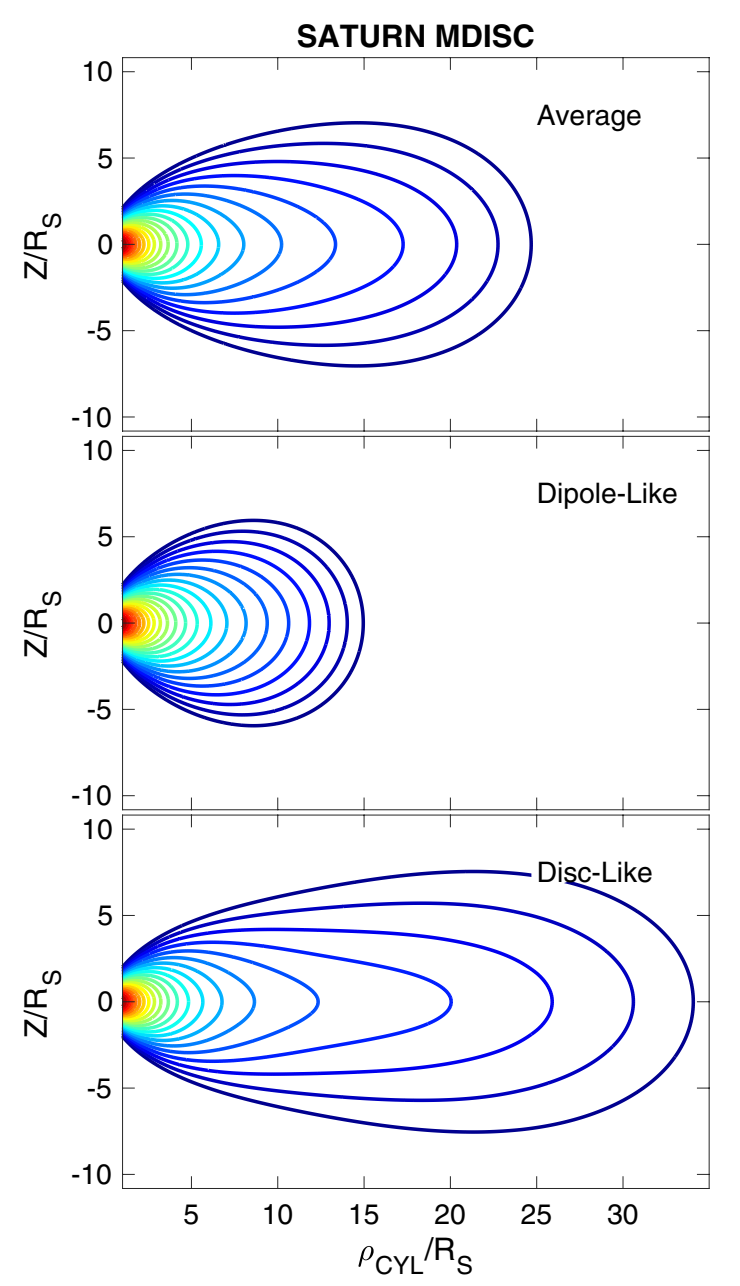

Fig. 1. Magnetic field lines for average, compressed and expanded configurations of the Kronian magnetodisc. Field lines in all panels are connected to the same ionospheric colatitudes. For this and other Figures, $\rho_{\mathrm{CYL}}$ denotes cylindrical radial distance, and $Z$ denotes vertical distance from the equator.

characteristic distance at which the model field strength equals that of the pure dipole moves further outwards as the system expands.

The equatorial segment of the current density distributions which produce the magnetic field structures, shown in Figures 1 and 2, are plotted in Figure 3. Evidently the most intense disc currents correspond to the most expanded configuration. The expanded configuration's outer magnetosphere is also dominated by inertial current (labelled "J $\mathrm{CENTRIF}_{\text {") }}$ associated with the centrifugal force on the subcorotating disc plasma.

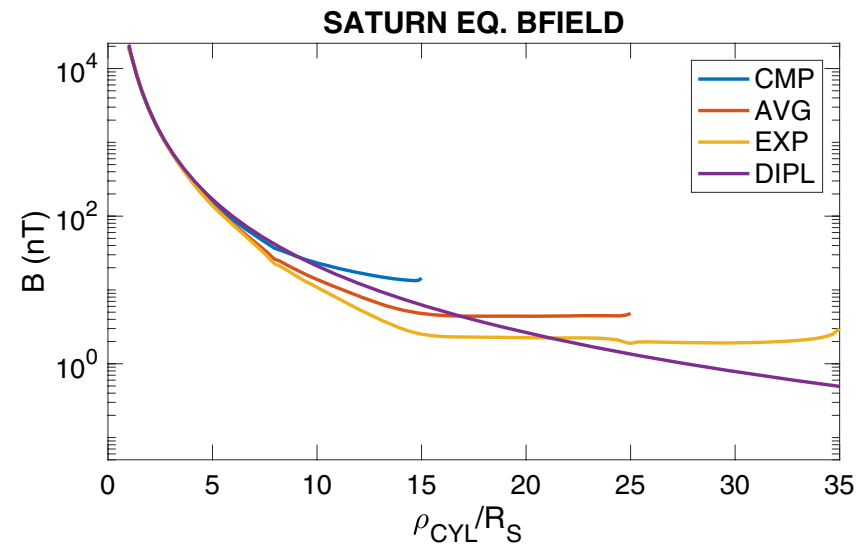

Fig. 2. Magnetic field strength profiles for the equatorial plane of the Kronian magnetodisc models. A pure dipole profile appropriate for Saturn is also shown for comparison.

All configurations show a "core" magnetospheric region at distances $\lesssim 10 R_{\mathrm{S}}$ for which current profiles are relatively insensitive to system size. The currents mostly flow in the positive sense (i.e., parallel to local corotation with the planet) however, layers of negative current are also seen at the inner edges of the thermal and suprathermal plasma discs. These arise in order to maintain overall force balance in a region where the pressure gradient force is directed inward (towards) the planet, rather than outward. The current profiles labelled " $\mathrm{J}_{\mathrm{HOT}}$ " and "J $\mathrm{COLD}_{\mathrm{C}}$ " are respectively associated with that part of the total current which balances local pressure gradient forces for the hot and cold plasma populations. We see that all current profiles are comparable in the outer magnetosphere for the most compressed system. Achilleos et al. (2010b) demonstrated that the parameter $K_{h}$ could be used to characterise realistic global changes in the hot plasma content of the magnetosphere. For Saturn, Cassini plasma data indicate that these changes can cause the hot pressure-related current to become the dominant contribution in the outer magnetosphere (see also e.g., Sergis et al., 2007, 2009).

More recently, Sorba et al. (2017) used Kronian magnetodisc models of this nature to examine the compressibility of Saturn's magnetosphere. The results presented in that study can be summarised by the following fit to the model results, which describes the variation of the magnetopause location $R_{\mathrm{mp}}$ as a function of the upstream solar wind dynamic pressure, $P_{\text {sw }}$, and the hot plasma parameter $K_{h}$ :

$$
\log R_{\mathrm{mp}}=a \log P_{\mathrm{sw}}+b \log K_{h}+c,
$$

where $R_{\mathrm{mp}}, P_{\mathrm{sw}}$ and $K_{h}$ are respectively expressed in units of $R_{\mathrm{S}}, \mathrm{nPa}$ and $\mathrm{Pa} \mathrm{m} \mathrm{T}{ }^{-1}$. 


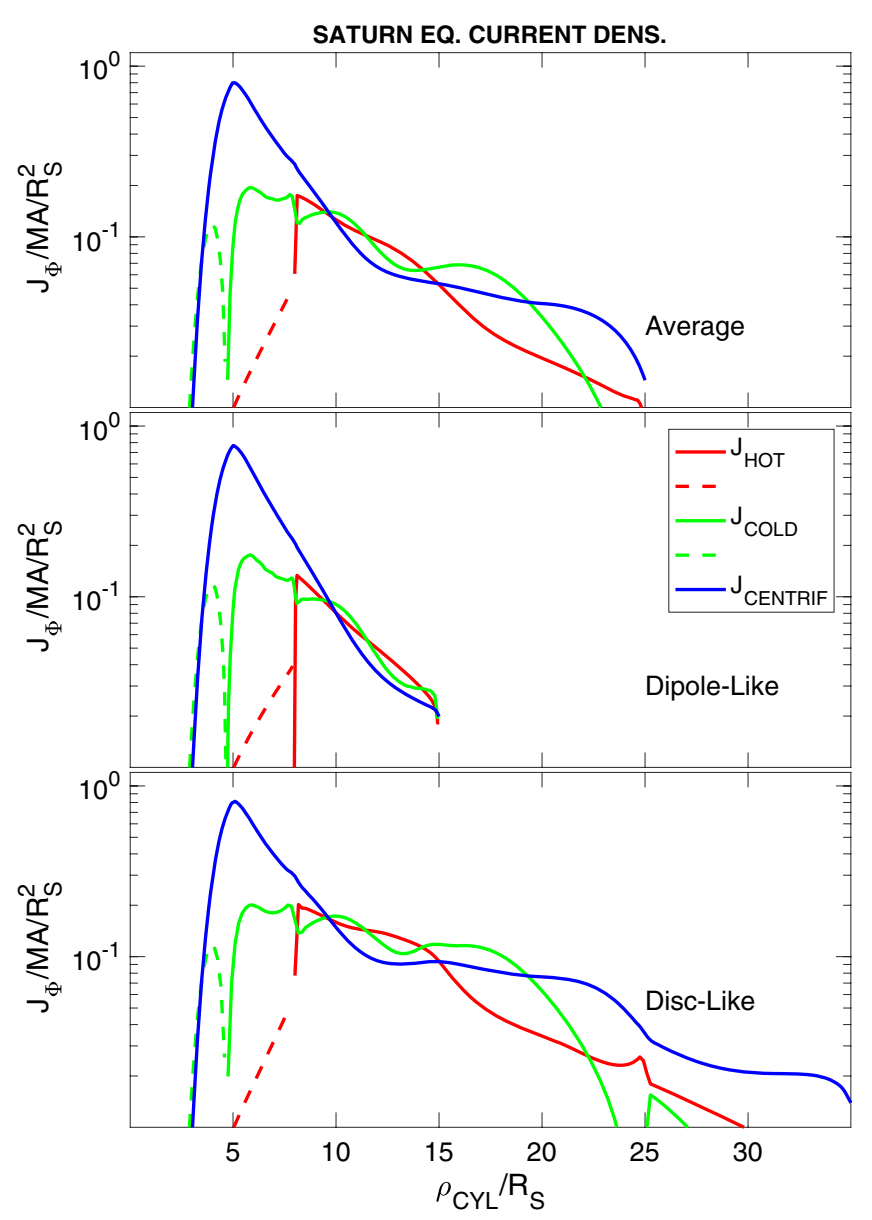

Fig. 3. Equatorial current density profiles of the Kronian magnetodisc models. Contributions associated with different forces on the plasma are colour-coded. Magnitude of current density is plotted (in units of mega-Ampères per squared planetary radius), with dashed curves indicating where current flows against the direction of planetary corotation.

Table 2. Fitted coefficients from equation (3), along with uncertainties $\sigma$ (95\% confidence), and root-mean-square (RMS) residuals (in $\log R_{\mathrm{mp}}$ ) relative to the results of Sorba et al. (2017).

\begin{tabular}{lccccccc}
\hline $\begin{array}{l}R_{\mathrm{mp}} \\
\text { regime }\end{array}$ & $a$ & $\sigma_{a}$ & $b$ & $\sigma_{b}$ & $c$ & $\sigma_{c}$ & $\begin{array}{c}\text { RMS } \\
\text { residual }\end{array}$ \\
\hline$\leq 25 R_{\mathrm{S}}$ & -0.228 & 0.010 & 0.094 & 0.009 & 0.99 & 0.14 & 0.052 \\
$>25 R_{\mathrm{S}}$ & -0.222 & 0.018 & 0.105 & 0.014 & 0.88 & 0.27 & 0.050 \\
All & -0.236 & 0.005 & 0.103 & 0.007 & 0.84 & 0.11 & 0.052 \\
\hline
\end{tabular}

The fitted coefficients $a, b$ and $c$ are given in Table 2 for three different cases: (i) "Compressed regime", i.e. using only the calculations from Sorba et al. (2017) for which $R_{\mathrm{mp}} \leq 25 R_{\mathrm{S}}$; (ii) "Expanded regime" $\left(R_{\mathrm{mp}}>25 R_{\mathrm{S}}\right)$; (iii) "Total regime" (14 $\left.R_{\mathrm{S}} \leq R_{\mathrm{mp}} \leq 40 R_{\mathrm{S}}\right)$, using all results from Sorba et al. (2017). We present these results here in order to indicate a convenient means for estimation of, for example, solar wind dynamic pressure corresponding to particular values of $R_{\mathrm{mp}}$ (system size) and $K_{h}$ (internal hot plasma state). A comprehensive analysis of the variation of the magnetospheric field

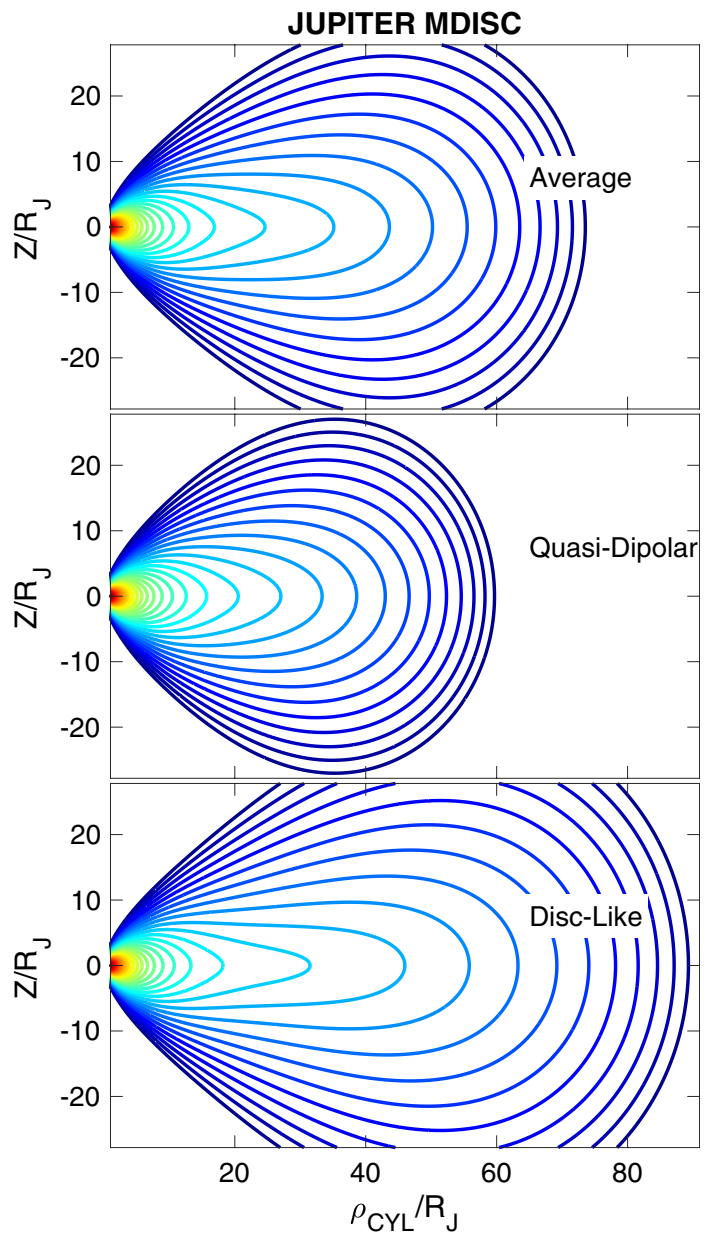

Fig. 4. Magnetic field lines for average, compressed and expanded configurations of the Jovian magnetodisc. Field lines in all panels are connected to the same ionospheric colatitudes.

structure and compressibility with these parameters is provided by Sorba et al. (2017), to whom the reader is referred for further details.

\subsection{Jupiter models}

For our calculations pertaining to the Jovian system, we used a grid with $N_{\mu}=501$ (as for the Saturn calculations), and $N_{r}=400$. The value of hot plasma index we used was $K_{h}=3 \times 10^{7} \mathrm{~Pa} \mathrm{~m} \mathrm{~T}^{-1}$, in accordance with the original magnetodisc model of Caudal (1986). We followed the same prescription for constructing equatorial plasma parameters as outlined in that study, but we refer the reader also to the more recent study of Nichols et al. (2015), who used more recent spacecraft data in a Jovian magnetodisc model which also incorporates pressure anisotropy.

The magnetic field lines of the Jovian models are shown in Figure 4, in a similar format to the Saturn results. Even for the most compressed configuration, the field contains some semblance of a "disc-like" character in the middle magnetosphere, and hence is labelled "quasi-dipolar". In fact, observations confirm that Saturn's magnetodisc radial field component can be more significantly reduced by the action of magnetospheric 


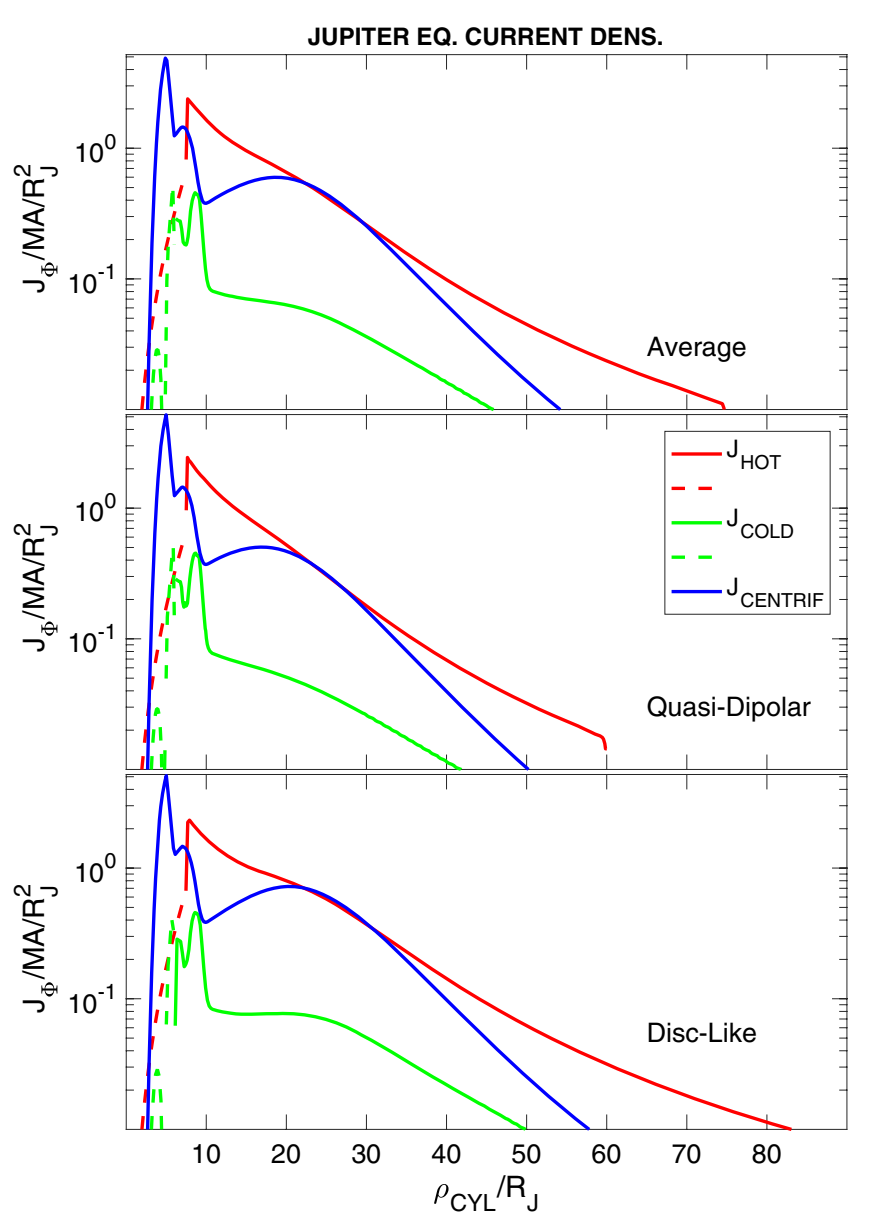

Fig. 5. Equatorial current density profiles of the Jovian magnetodisc models. Contributions associated with different forces on the plasma are colour-coded. Magnitude of current density is plotted (in units of mega-Ampères per squared planetary radius), with dashed curves indicating where current flows against the direction of planetary corotation.

compression, when compared to the Jovian system (see e.g., Arridge et al., 2008). We also note a distinct transition in the Jovian field from the very "disc-like" middle magnetosphere to field lines with much larger radii of curvature in the outer magnetospheric layer. These outer field lines are not dipolar in shape, but do represent the response of the plasma and field to the proximity and "bracing" effect of the prescribed model boundary (magnetopause).

In Figure 5, we show the analogous equatorial current profiles for the Jovian models. As for the case of Saturn, we can identify a rigid "core" magnetosphere, here within $\lesssim 20 R_{\mathrm{J}}$, where the current and field structure are relatively resistant to change in system size. The outer magnetosphere is clearly dominated by the hot pressure-related current for all configurations, but again we refer the reader to Nichols et al. (2015) for a more comprehensive study of the dominant current component in the outer Jovian magnetosphere.

Finally, in Figure 6, we show the distribution of total (hot + cold) plasma pressure for the different Jovian magnetodisc configurations. Note that the pressure is approximately uniform along field lines, as it is often dominated by the hot

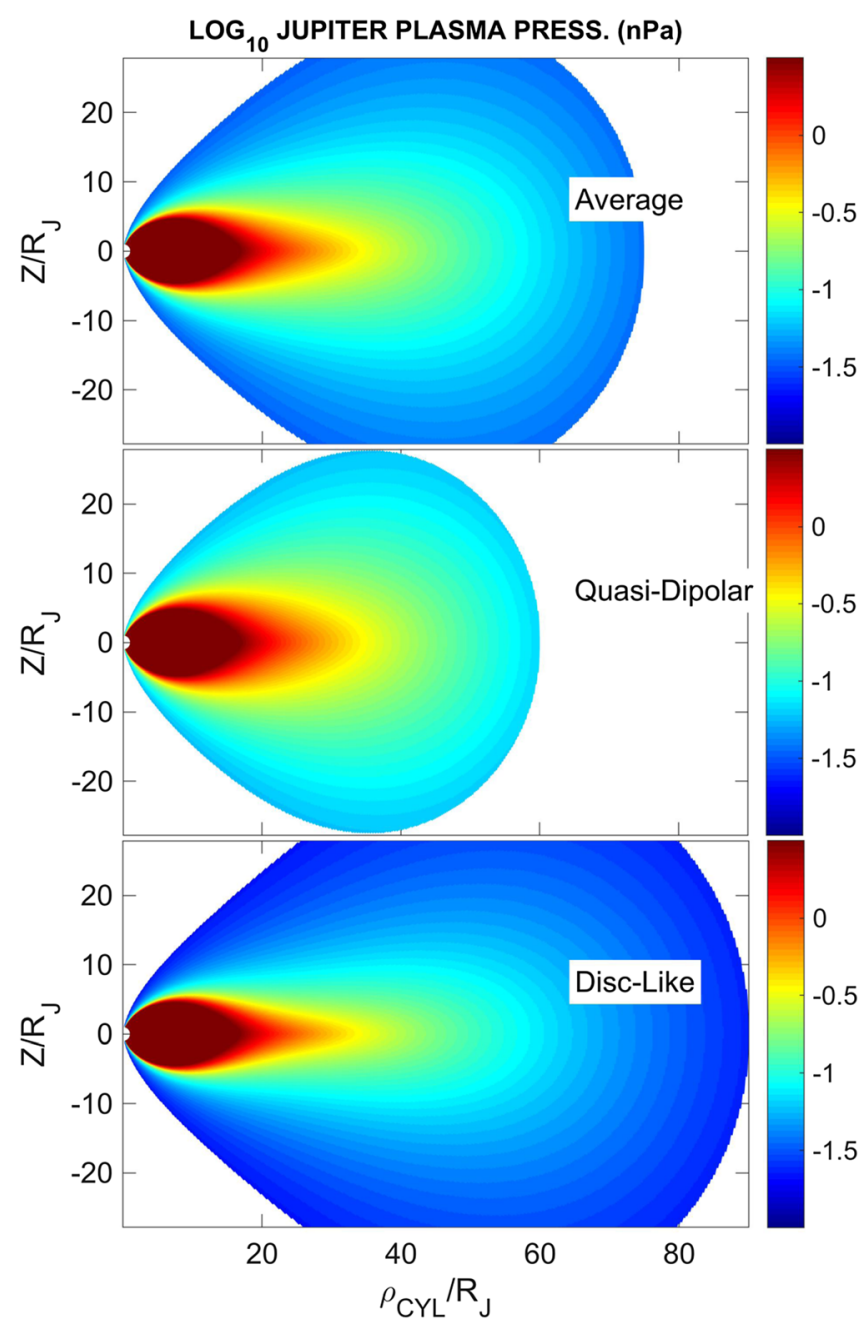

Fig. 6. Plasma pressure distributions (logarithmic) of the Jovian magnetodisc models.

plasma population, for which this property is assumed in the modelling procedure. Note also that the model assumes the presence of plasma only on closed field lines, and this is reflected in the absence of pressure contours in the "polar cap" regions of the model. The most expanded configuration displays the lowest pressure values in the outer magnetosphere, since flux tubes with a given cross-section in magnetic flux are spread over a wider volume, thus lowering the pressure of the plasma which they contain.

\section{The Europlanet PSWS MAGNETODISC service}

The models used in this study were produced by the same calculation method as those model outputs presently available via the VESPA interface (see Section 2). The Planetary Space Weather Services (PSWS, http://planetaryspaceweathereuroplanet.irap.omp.eu/, André et al., 2018) of the Europlanet 2020-RI project (http://www.europlanet-2020-ri.eu/) aims at developing services for predicting the solar wind properties 
following solar perturbations throughout the Solar System, as well as modelling how the space environment of the various planets is affected by the solar wind. In this context, we have developed the PSWS MAGNETODISC service which is a web interface allowing online runs on request of the UCL Magnetodisc Model described here. The service is publicly available and operational since September 2018. The user interface has been conceived to be easy to use and intuitive, and can be found on the tab entitled "Request Run". Users of MAGNETODISC can thus prescribe the parameters $R_{\mathrm{mp}}$ and $K_{h}$, described earlier in the paper, in order to receive a corresponding magnetodisc model for the planet of their choice.

Thus, the MAGNETODISC service enables the user a "finer" exploration of that parameter space than, for example, the archive of model outputs provided through VESPA (http://www.europlanet-vespa.eu/, Erard et al., 2018). When the results are ready, the user will receive an email with a link to the web page listing all the past requested runs. All the runs are freely available, with the more recent ones at the top of the page. Each run is briefly described in one line and the user can click on two different icons at the right-hand side of the line: one for downloading the run results as a zipped file in CDF format, and one to send the result of the simulation via Simple Application Messaging Protocol (SAMP, Taylor et al., 2015) to VO tools for rapid visualization. Before this operation, the user needs to be first connected to a hub. The MAGNETODISC service can be used in order to compare model outputs with the new observations obtained by the NASA Juno spacecraft at Jupiter, as well as with the past observations of Cassini at Saturn. The service will also be useful in order to help with the preparation for the ESA JUpiter ICy moon Explorer (JUICE) mission. Future developments include the linkage of the MAGNETODISC service with the HELIOPROPA service (http://heliopropa.irap.omp.eu/) which will be used to predict, from a one-dimensional magnetohydrodynamic propagation model, the time-dependent dynamic pressure of the solar wind upstream of Jupiter and Saturn and prescribe the corresponding parameter $R_{\mathrm{mp}}$ for the runs on request.

\section{Discussion and conclusions}

The force-balance method described herein, used to provide magnetic field and plasma models for the disc-like magnetospheres of Jupiter and Saturn via the PSWS MAGNETODISC service, produces a wide variety of structures in response to changes in system size, equivalent to changes in solar wind dynamic pressure upstream of the relevant magnetosphere (for a given value of the internal plasma parameter $K_{h}$ ). Such models are thus useful for characterising the changes in plasma and field conditions in response to what may be referred to as "space weather" events at the orbits of these gas giant worlds. The versatility of a combined field-plus-plasma model has also been useful for studying the physical origin of the change in field structure and current distributions in the magnetosphere, in terms of the various forces exerted on the plasma.

We have also presented a convenient formula for estimating the effect on system size $R_{\mathrm{mp}}$ for Saturn in response to changes in both solar wind dynamic pressure and to changes in internal hot plasma content - thus building significantly on traditional studies of magnetospheric compressibility which focussed solely on solar wind-driven changes. The advent of the Cassini mission has enabled us to make this advancement in how we parameterise magnetospheric behaviour (see e.g., Achilleos et al., 2008; Pilkington et al., 2015; Sorba et al., 2017 and references therein). We have also provided an introduction for potential users of the PSWS MAGNETODISC service, whose output is illustrated by the reference models we have presented.

Note that the magnetodisc models described herein, although based on magnetostatic equilibrium, have also facilitated studies of the influence of the pressure of a "hot" or energetic particle population - an aspect which is not usually included in standard MHD magnetospheric models.

Acknowledgements. NA and PG were supported by UK STFC grant ST/N000722/1 and the European Planetary Network project. AMS was supported by a UK STFC studentship. The editor thanks Nick Sergis and an anonymous referee for their assistance in evaluating this paper.

\section{References}

Achilleos N, Arridge CS, Bertucci C, Jackman CM, Dougherty MK, Khurana KK, Russell CT. 2008. Large-scale dynamics of Saturn's magnetopause: Observations by Cassini. J Geophys Res 113: A11209. DOI: 10.1029/2008JA013265.

Achilleos N, Guio P, Arridge CS. 2010a. A model of force balance in Saturn's magnetodisc. Mon Not Royal Ast Soc 401: 2349-2371. DOI: 10.1111/j.1365-2966.2009.15865.x. arXiv:0909.1514.

Achilleos N, Guio P, Arridge CS, Sergis N, Wilson RJ, Thomsen MF, Coates AJ. 2010b. Influence of hot plasma pressure on the global structure of Saturn's magnetodisk. Geophys Res Lett 372: L20201. DOI: 10.1029/2010GL045159. arXiv:1008.3791.

André N, Grande M, Achilleos N, Barthélémy M, Bouchemit M, et al. 2018. Virtual planetary space weather services offered by the Europlanet H2020 research infrastructure. Planet Space Sci 150: 50-59. DOI: 10.1016/j.pss.2017.04.020.

Arridge CS, Russell CT, Khurana KK, Achilleos N, Cowley SWH, Dougherty MK, Southwood DJ, Bunce EJ. 2008. Saturn's magnetodisc current sheet. J Geophys Res 113: A04214. DOI: 10.1029/2007JA012540.

Bunce EJ, Cowley SWH, Alexeev II, Arridge CS, Dougherty MK, Nichols JD, Russell CT. 2007. Cassini observations of the variation of Saturn's ring current parameters with system size. $J$ Geophys Res 112: A10202. DOI: 10.1029/2007JA012275.

Caudal G. 1986. A self-consistent model of Jupiter's magnetodisc including the effects of centrifugal force and pressure. J Geophys Res 91: 4201-4221. DOI: 10.1029/JA091iA04p04201.

Connerney JE, Acuña MH, Ness NF. 1981a. Modeling the jovian current sheet and inner magnetosphere. J Geophys Res 86: 8370-8384. DOI: 10.1029/JA086iA10p08370.

Connerney JEP, Acuna MH, Ness NF. 1981b. Saturn's ring current and inner magnetosphere. Nature 292: 724-726. DOI: 10.1038/ $292724 \mathrm{a} 0$.

Erard S, Cecconi B, Le Sidaner P, Rossi AP, Capria MT, et al. 2018. VESPA: A community-driven Virtual Observatory in Planetary Science. Planet Space Sci 150: 65-85. DOI: 10.1016/j.pss.2017. 05.013. arXiv:1705.09727.

Giampieri G, Dougherty M. 2004. Modelling of the ring current in Saturn's magnetosphere. Ann Geophys 22: 653-659. DOI: 10.5194/angeo-22-653-2004. 
Joy SP, Kivelson MG, Walker RJ, Khurana KK, Russell CT, Ogino T. 2002. Probabilistic models of the Jovian magnetopause and bow shock locations. J Geophys Res (Space Phys) 107(A10): 1309. DOI: 10.1029/2001JA009146.

Kellett S, Bunce EJ, Coates AJ, Cowley SWH. 2009. Thickness of Saturn's ring current determined from north-south Cassini passes through the current layer. J Geophys Res 114: A04209. DOI: 10.1029/2008JA013942.

Lackner K. 1970. Deformation of a magnetic dipole field by trapped particles. J Geophys Res 75: 3180-3192. DOI: 10.1029/ JA075i016p03180.

Nichols JD, Achilleos N, Cowley SWH. 2015. A model of force balance in Jupiter's magnetodisc including hot plasma pressure anisotropy. J Geophys Res (Space Phys) 120(A9): 10. DOI: 10.1002/2015JA021807.

Pilkington NM, Achilleos N, Arridge CS, Guio P, Masters A, Ray LC, Sergis N, Thomsen MF, Coates AJ, Dougherty MK. 2015. Internally driven large-scale changes in the size of Saturn's magnetosphere. J Geophys Res (Space Phys) 120: 7289-7306. DOI: 10.1002/2015JA021290.

Sergis N, Krimigis SM, Mitchell DG, Hamilton DC, Krupp N, Mauk BM, Roelof EC, Dougherty M. 2007. Ring current at Saturn:
Energetic particle pressure in Saturn's equatorial magnetosphere measured with Cassini/MIMI. Geophys Res Lett 34: L09102. DOI: 10.1029/2006GL029223.

Sergis N, Krimigis SM, Mitchell DG, Hamilton DC, Krupp N, Mauk BH, Roelof EC, Dougherty MK. 2009. Energetic particle pressure in Saturn's magnetosphere measured with the Magnetospheric Imaging Instrument on Cassini. J Geophys Res 114: A02214. DOI: 10.1029/2008JA013774.

Sorba AM, Achilleos NA, Guio P, Arridge CS, Pilkington NM, Masters A, Sergis N, Coates AJ, Dougherty MK. 2017. Modeling the compressibility of Saturn's magnetosphere in response to internal and external influences. J Geophys Res (Space Phys) 122: 1572-1589. DOI: 10.1002/2016JA023544.

Sozou C, Windle DW. 1970. The effect of a large ring current on the topology of the magnetosphere. Planet Space Sci 18: 699-707. DOI: 10.1016/0032-0633(70)90051-6.

Taylor MB, Boch T, Taylor J. 2015. SAMP: the Simple Application Messaging Protocol: Letting applications talk to each other. Astron Comput 11: 81-90. DOI: 10.1016/j.ascom.2014.12.007. arXiv:1501.01139.

Cite this article as: Achilleos N, Guio P, André N \& Sorba AM 2019. A magnetodisc model service for planetary space weather studies. $J$. Space Weather Space Clim. 9, A24. 